\title{
O direito à saúde e o papel do Sistema Único de Saúde em tempos de pandemia no
}

\section{Brasil}

\author{
The right to health and the role of the Unified Health System in times of pandemic in Brazil \\ EI derecho a la salud y el papel del Sistema Único de Salud en tiempos de pandemia en Brasil
}

Recebido: 07/07/2021 | Revisado: 12/07/2021 | Aceito: 08/09/2021 | Publicado: 11/09/2021

\author{
João Marcos Batista Gomes de Araujo \\ ORCID: https://orcid.org/0000-0001-6877-2179 \\ Faculdade Santa Maria, Brasil \\ E-mail: joaomarcosbg@hotmail.com \\ Adryele Gomes Maia \\ ORCID: https://orcid.org/0000-0002-7433-7138 \\ Universidade Federal do Rio Grande do Norte, Brasil \\ E-mail: adryelegm@gmail.com \\ Francyely dos Santos Moura \\ ORCID: https://orcid.org/0000-0003-3791-2049 \\ Universidade Federal do Rio Grande do Norte, Brasil \\ E-mail: mourafrancyely@gmail.com \\ Micael Pyerre Martins Duarte \\ ORCID: https://orcid.org/0000-0002-0424-8244 \\ Universidade Federal do Rio Grande do Norte, Brasil \\ E-mail: micaelpmduarte@gmail.com \\ Maiara Bezerra Dantas \\ ORCID: https://orcid.org/0000-0002-0389-6792 \\ Universidade Federal do Rio Grande do Norte, Brasil \\ E-mail: maiara-dantas13@hotmail.com \\ Deyse Janiele Bernardo Oliveira \\ ORCID: https://orcid.org/0000-0002-1270-8431 \\ Universidade Federal do Rio Grande do Norte, Brasil \\ E-mail: deyse.oliveiira@hotmail.com \\ José Cândido da Silva Nóbrega \\ ORCID: https://orcid.org/0000-0002-0976-3763 \\ Universidade Federal de Campina Grande, Brasil \\ E-mail: jcandidosn@uol.com.br \\ José Djalisson Santos Oliveira \\ ORCID: https://orcid.org/0000-0002-3335-1144 \\ Centro Universitário de João Pessoa, Brasil \\ E-mail: oliveira.eujose@gmail.com
}

\begin{abstract}
Resumo
No Brasil o direito a saúde, está consagrado na Constituição Federal de 1988 como um direito social fundamental, e por conseguinte, recebe total proteção jurídica, e foi a partir da criação do Sistema Único de Saúde (SUS), que se intensificou o debate sobre o financiamento da política de saúde por parte de gestores, e entidades, por considerar a estrutura insuficiente para atender a demanda dos cidadãos brasileiros. Dessa maneira, o presente artigo tem como objetivo analisar a saúde pública no Brasil a partir de uma perspectiva histórico-crítica, tendo como base a Carta Constitucional 1988, a Lei n ${ }^{\circ} 8.080$ e a EC/95 que regulamentam as ações e orçamento do SUS debruçando-se em observar como essas leis são aplicadas na atualidade e se conseguem suprir as necessidades e demandas da população. Para a realização da pesquisa empregou-se o método de abordagem dedutivo, partindo da análise da lei constitucional, para sua aplicação prática no contexto da pandemia. Foi realizada uma análise histórico-evolutivo. O nível de profundidade desse estudo foi construído através de uma pesquisa exploratória, os dados foram coletados através da pesquisa bibliográfica e documental. Diante das reflexões estudadas ao longo da pesquisa, foi possível constatar que, apesar dos direitos fundamentais estarem consolidados na Carta Magna, verifica-se que o Estado como garantidor dos direitos fundamentais, não garante o suficiente para que se possa efetivar totalmente essas garantias geradas principalmente pela aplicação insuficiente de recursos e pela falta de atendimento adequado do sistema durante a crise agravada pela pandemia instalada no Brasil.
\end{abstract}

Palavras-chave: Constituição Federal; Direitos fundamentais; Pandemia. 


\begin{abstract}
In Brazil, the right to health is enshrined in the Federal Constitution of 1988 as a fundamental social right, and therefore, receives full legal protection, and it was with the creation of the Sistema Único de Saúde (SUS) that the debate on the financing of health policy intensified by managers and entities, considering the structure insufficient to meet the demand of Brazilian citizens. Thus, this article aims to analyze public health in Brazil from a historicalcritical perspective, based on the Constitutional Charter 1988, Law $\mathrm{n}^{\circ} 8080$ and EC/95, which regulate the actions and budget of the SUS. if in observing how these laws are applied nowadays and if they manage to supply the population's needs and demands. To carry out the research, the deductive approach method was used, starting from the analysis of the constitutional law, for its practical application in the context of the pandemic. A historical-evolutionary analysis was carried out. The level of depth of this study was built through an exploratory research, the data were collected through bibliographical and documentary research. In view of the reflections studied throughout the research, it was possible to see that, despite the fundamental rights being consolidated in the Magna Carta, it appears that the State as guarantor of fundamental rights does not guarantee enough to fully implement these guarantees generated mainly by the insufficient application of resources and by the lack of adequate system service during the crisis aggravated by the pandemic in Brazil.
\end{abstract}

Keywords: Federal Constitution; Fundamental rights; Pandemic.

\title{
Resumen
}

En Brasil, el derecho a la salud está consagrado en la Constitución Federal de 1988 como un derecho social fundamental y, por lo tanto, recibe plena protección legal, y fue con la creación del Sistema Único de Saúde (SUS) que se intensificó el debate sobre el financiamiento de la política de salud por parte de los gestores. y entidades, considerando la estructura insuficiente para satisfacer la demanda de los ciudadanos brasileños. Así, este artículo tiene como objetivo analizar la salud pública en Brasil desde una perspectiva histórico-crítica, con base en la Carta Constitucional de 1988, Ley n 8080 y EC/95, que regulan las acciones y el presupuesto del SUS. si en observar cómo se aplican estas leyes en la actualidad y si logran suplir las necesidades y demandas de la población. Para la realización de la investigación se utilizó el método de enfoque deductivo, a partir del análisis del derecho constitucional, para su aplicación práctica en el contexto de la pandemia. Se realizó un análisis histórico-evolutivo. El nivel de profundidad de este estudio se construyó a través de una investigación exploratoria, los datos fueron recolectados a través de una investigación bibliográfica y documental. A la vista de las reflexiones estudiadas a lo largo de la investigación, se pudo constatar que, a pesar de que los derechos fundamentales están consolidados en la Carta Magna, parece que el Estado como garante de los derechos fundamentales no garantiza lo suficiente para implementar plenamente estas garantías generadas principalmente por la insuficiente aplicación de recursos y por la falta de un adecuado servicio del sistema durante la crisis agravada por la pandemia en Brasil.

Palabras clave: Constituicion Federal; Derechos fundamentales; Pandemia.

\section{Introdução}

O presente estudo trata sobre a análise da aplicação do direito a saúde e das garantias constitucionais, no que tange a prestação positiva do estado através da efetivação do SUS e da sua assistência nesse período de pandemia da SARS-CoV-2. No Brasil o direito a saúde não era considerado um direito de todos, vindo a ganhar status de direito fundamental a partir da promulgação da Constituição Federal de 1988.

Em relação a isso, a relevância dessa pesquisa é demonstrada através do estudo dos impactos do direito à saúde, desde sua efetivação até o período atual da pandemia, em vista disso, o escopo desse estudo é analisar a efetivação e aplicação do direito a saúde no Brasil atingindo seu sentido material.

Ademais, apesar da grande importância da criação do Sistema Único de Saúde e devido a sua complexidade, além da sua positivação na Constituição, foi preciso organizar o seu funcionamento através da Lei n 8.080/1990 em que se deu o seu nascimento e se efetivou as condições para que ocorresse a proteção, recuperação da saúde, organização e o funcionamento dos serviços essenciais. 
Diante dessa conjectura, surge a preocupação em saber se a Carta Constitucional, que traz em seu escopo o direito a saúde como um direito social e fundamental, consegue garantir uma assistência igualitária aos usuários diante desse contexto da pandemia através da utilização do SUS?

Haja vista a essa problemática circundante, essa pesquisa tem como objetivo geral: analisar a saúde pública no Brasil a partir de uma perspectiva histórico-crítica, tendo como base a CF/88, a Lei n 8.080 e a EC/95 que regulamentam as ações e orçamento do Sistema de Saúde, debruçando-se em observar se a partir da aplicação dessas leis, será possível entender se elas conseguem suprir as necessidades e demandas da população que são assistidas por esse Sistema, bem como a aplicação dos recursos públicos, as parcerias e formas de financiamento voltadas para o SUS nesse período pandemia.

Buscando atingir os fins propostos, o presente artigo foi elaborado através do método de abordagem dedutivo, partindo da análise da aplicação da lei na Carta Constitucional, analisando sua aplicação prática no contexto da pandemia. Desse modo, será realizada uma análise histórico-evolutivo partindo da construção do Sistema Único e os elementos fundamentais de sua legislação, visando capturar seus efeitos e entender como são compreendidos no sistema atual, tendo em vista sua latente necessidade de aplicação nesse período de pandemia. Em nível de profundidade, esse estudo foi construído através de uma pesquisa exploratória, a coleta de dados foi bibliográfica através de teses, dissertações, livros e artigos e a pesquisa documental partindo da análise legislativa.

Para melhor compreensão essa pesquisa organiza-se primeiramente em fazer uma abordagem do direito a saúde na Carta Magna, se faz necessário também, fazer uma análise do direito a saúde como garantia fundamental, por conseguinte será feita uma breve abordagem de como se deu à criação do SUS no Brasil, a posteriori será verificado como funciona alguns pontos relevantes do financiamento desse Sistema, e por fim, será feito uma síntese de como se dá seu funcionamento nesse período de pandemia no Brasil.

\section{O direito a Saúde na Constituição Federal de 1988}

O direito a saúde, conforme descrito na Carta Magna, está estabelecido no título dos direitos sociais, e teve um histórico de várias mudanças, o qual adquiriu reconhecimento no tocante a sua grande importância, e ao analisarmos o contexto evolutivo, constata-se que na constituição de 1934 a saúde possuía status de direito do trabalhador e possuía caráter privado, enquanto que na constituição de 1937 a preocupação maior era a saúde das crianças, já na constituição de 1946 a saúde era tratada como mera divisão de regras da unidade federativa (Araujo, 2008).

Entre tanto, com a promulgação da Constituição, houve uma dilatação desta garantia quando a saúde foi declarada como direito social, de $2^{a}$ dimensão, com a transição do estado liberal para um estado social no qual a saúde passou a ser vista também como um direito fundamental ( $1^{\mathrm{a}}$ dimensão), ou seja, para a pessoa ter direito a uma vida digna ela necessita de saúde. Pode-se constatar também que a saúde transita pelos direitos de $3^{\text {a }}$ Dimensão, pois, é um direito que visa a proteção da coletividade. (Bahia, 2012).

O direito à saúde foi incorporado, no título da ordem social, e tem como objetivos o bem-estar e a justiça social, e dentro dessa perspectiva, o artigo $6^{\circ}$ da constituição, considera a educação, a saúde, alimentação, a moradia, o lazer, a segurança, trabalho, a previdência social, a proteção da maternidade e a infância e a assistência aos desamparados como direitos sociais básicos (BRASIL, 1988).

Em complemento, o artigo 196 da Carta Maior, afirma que o direito a saúde é um direito pertencente à todos e deve ser provido pelo Estado, o qual deve ser garantido através de políticas sociais e econômicas que viabilizem a diminuição do risco de doenças e garanta um acesso igualitário e universal a todos usuários (BRASIL, 1988). 
Seguindo o pensamento de Moura (2013), o direito à saúde foi eleito dentro dos direitos sociais pelo constituinte como de peculiar importância, demonstrando essa importância, através da forma como foi tratada em capítulo próprio, sendo perceptível o cuidado que se teve com esse bem jurídico. E com efeito, o direito à saúde, por estar intimamente atrelado ao direito à vida, manifesta a proteção constitucional à dignidade da pessoa humana.

Em síntese, através do contexto percebe-se que, o direito a saúde apesar de ser um direito social foi consagrado na Carta Magna com relevante interesse do constituinte, pois trata-se de um direito fundamental que passou por uma série de lutas e conquistas e que apesar de ser protegido pela Constituição ainda precisa passar por uma série de mudanças e investimentos para suprir sua demanda e ser distribuído de forma igualitária entre a população brasileira.

\section{A Saúde como Direito Fundamental e a Criação do SUS}

O direito à saúde, está consagrado na Constituição Federal de 1988, como um direito social fundamental e desse modo, recebe total proteção na ordem jurídica brasileira e ao ser reconhecido como direito social fundamental, o estado obrigou-se a prestações positivas e por consequência foram formuladas politicas publicas sociais e econômicas destinadas a promoção e recuperação da saúde pública (Moura, 2013).

Essas prestações positivas em que se obriga o Estado, possuem grande importância que ultrapassam o campo da discricionaridade administrativa, e deve ser inafastável uma proteção constitucional de tal modo que, as pautas que estão ligadas a atuação governamental, jamais poderão ser relegadas a conceitos de oportunidade ou conveniência do agente público, eis que não podem transformar-se em mero jogo de palavras visto, que são indispensáveis à manutenção do "status" de dignidade da pessoa humana. (Bahia, 1999).

Nesta senda, ainda referente ao direito a saúde, foram inseridos no texto Constitucional importantes matizes da dimensão organizatória e procedimental, e nessa perspectiva, os artigos 198 e 200 atribuíram ao Sistema Único de Saúde a coordenação e execução das políticas para a promoção e proteção da saúde no Brasil.

Além da previsão constitucional da criação de uma estrutura organizacional para garantir o direito a saúde, a Carta constitucional, também indicou como seria a atuação desse órgão e quais deveriam ser seus objetivos conferindo o esboço do que seria do SUS, entretanto, apesar dessa previsão os procedimentos de funcionamento e as atribuições do órgão só puderam ser concretizadas a partir da elaboração de leis especificas (Moura, 2013).

E com esse propósito, a Lei Federal 8.080, de 19 de setembro de 1990, foi criada e dispõem sobre as atribuições e funcionamento do Sistema Único de Saúde, bem como a Lei Federal n 8142 de dezembro de 1990, que trata sobre a participação da comunidade na gestão do sistema único de saúde e sobre as transferências intergovernamentais e de recursos financeiros da área de saúde.

Diante do exposto, fica a cargo do governo direcionar sua atuação para programar as políticas públicas que são indispensáveis para resolver as necessidades sociais, e desta feita é consonante com o comprometimento de uma vida digna, à criação do Sistema Único de Saúde, integra "Ações e serviços públicos a uma rede regionalizada e hierarquizada para fins de dar continuidade à política de saúde social” art. 198 (Brasil, 1988).

Apesar da grande importância da criação desse Sistema de saúde e devido a sua complexidade, foi preciso organizar seu funcionamento através da Lei $n^{\circ} 8.080$ de 19 de setembro de 1990 onde se deu a criação do SUS, e se efetivou as condições para que ocorresse a proteção, recuperação da saúde, organização e o funcionamento dos serviços essenciais, entretanto não obstante a estar positivado, esse sistema ainda se defronta com algumas dificuldades como no caso em exposição à insuficiência de recursos. 


\section{A Crise do Financiamento do SUS}

Até a promulgação da Carta Magna, o direito a saúde não tinha sido reconhecido como um direito fundamental, ou seja, não era um direito de todos, portanto, quem tinha que arcar com os cuidados da saúde era o próprio indivíduo, ocasionalmente, as políticas que foram desenvolvidas pelo Estado eram bastante restritas, sendo que esse panorama era bastante corriqueiro nas primeiras décadas do século passado, por tudo isso o estado brasileiro restringia a atuação da saúde em ações de saneamento nas grandes cidades e portos e no combate a epidemias, principalmente quando estas epidemias interferiam na economia e traziam prejuízos.

Sabendo, portanto, da importância do direito a saúde e no que diz respeito ao seu financiamento, o art. 195, da CF/88, afirma que "a seguridade social será financiada por toda a sociedade, de forma direta e indireta, nos termos da lei, mediante recursos provenientes dos orçamentos da União, dos Estados, do Distrito Federal e dos Municípios”. Ou seja, a responsabilidade pelo financiamento do Sistema é de obrigação primária das três esferas de governo.

Além disso, no âmbito da União, havia a determinação de que 30\% do Orçamento da Seguridade Social (OSS), excluindo o seguro-desemprego, deveriam ser alocados ao SUS até que a lei de diretrizes orçamentárias fosse aprovada. Entretanto, essa regra não foi cumprida, tendo sido vetada em 1994. E como consequência, de acordo com a Organização Panamericana da Saúde (2013), a falta de recursos para financiamento dos serviços deflagrou uma crise importante no SUS e obrigou o Ministério da Saúde (MS) a recorrer a empréstimos junto ao Fundo de Amparo ao Trabalhador (Mendes \& Marques, 2010).

Em decorrência do estado de calamidade devido a pandemia, foram editados créditos extraordinários com ampliação de despesas não contabilizadas no teto de gasto previsto na Emenda Constitucional n ${ }^{\circ}$ 95, de 2016. A maior parte das despesas foi financiada por endividamento, viabilizado pela suspensão da regra de ouro que afeta a saúde, tendo em vista que a maior parte de suas despesas é corrente (Chioro et al., 2021).

Neste contexto, a EC/95, combinando teto declinante que reduz estruturalmente despesas públicas em relação ao tamanho da economia e piso congelado para a saúde, não é capaz de absorver a demanda crescente por ações e serviços públicos de saúde. Ante o exposto, é fundamental discutir novas regras fiscais capazes de financiar o SUS, tendo em vista as previsões constitucionais da universalidade e integralidade e os fatores estruturais e conjunturais associados à pandemia que impactam o sistema (Chioro et al., 2021).

Com a vigência da EC/95 a perda de recursos federais foi estimada em R \$ 22,5 bilhões entre 2018 e 2020 , sendo R\$ 13,6 bilhões somente em 2019. Isso revela o potencial de desfinanciamento do SUS pela EC 95 em comparação com a vinculação da aplicação mínima federal em ASPS dada pela EC 86 (Santos, 2020).

Dessa forma, é perceptível que apesar de uma série de medidas que garantem investimentos no sistema de saúde, os recursos não tem uma aplicação satisfatória devido as medidas efetivas que diminuem o teto dos gastos entre eles o da saúde através da EC/95, o qual se torna uma decisão contraditória pois, com o aumento da população e dos casos, durante essa pandemia população, também se aumenta a busca por serviços assistenciais de saúde.

\section{O Sistema Único de Saúde e a Realidade em Tempos de Pandemia da Covid-19 no Brasil}

No Brasil o SUS, é resultado de inúmeras lutas, contribuições, pesquisas, investimentos e outras medidas, e é apresentado como impossível de ser gerido e operado publicamente. Isto ocorre não apenas em meio à maior pandemia do presente século, dá-se em meio a inúmeras ações para transformar em mercadoria políticas públicas vistas pela população como direitos. Trata-se de uma das várias estratégias capitalistas, em sua etapa neoliberal, para lidar com mais uma de suas longas crises (Silva \& Ruiz, 2020). 
Atualmente, além da precarização vivida por esse sistema, ainda existe a pandemia mundial da pandemia, que é um vetor de demonstração, em que se verifica como o estado responde à ameaça frente a sua direção, pois apesar de haver um índice exorbitante de contágio, o Estado deve estar preparado para responder com a mínima lesão possível ao bem maior que é a vida, entretanto, o que acontece é a defasagem do Sistema Único de Saúde que se traduz na aparência pandêmica sem resposta e pela falta de fornecimento de estrutura um atendimento igualitário, demonstrando que o pensamento de esvaziamento real e simbólico dos direitos sociais está se concretizando (Maia, 2020).

Ao avançar sobre o Brasil, a pandemia gerou uma elevada demanda emergencial e crescente ao Sistema Único de Saúde (SUS), descortinando uma das dimensões mais graves da crise sanitária brasileira: a do seu componente hospitalar. A estrutura assistencial hospitalar brasileira historicamente insuficiente, geograficamente mal distribuída, irregularmente integrada aos sistemas locais e regionais, com indicadores de desempenho contestáveis, além de severamente desgastada pelo subfinanciamento crônico, vê-se então diante da impactante demanda por um grande número de leitos hospitalares (gerais e de Unidades de Terapia Intensiva - UTI) para o cuidado às vítimas da SARSCoV-2, sobretudo as mais graves (Campos \& Canabrava, 2020, p. 01).

Quando se fala na crise do SUS, esse não é problema atual, e sim um problema que perpétua desde o período pósconstituinte, e com o comprometimento da sustentabilidade econômica do programa, foi permitido abertura de capital estrangeiro, além do congelamento dos gastos públicos afetando o investimento na saúde (Paim, 2018).

À medida que avança a pandemia no Brasil, são expostos os investimentos insuficientes na saúde pública, o que significa que o acesso ao atendimento médico não está disponível para todos e expõe o fracasso do estado em planejar e prestar serviços que atendam às necessidades da população em geral (Araujo, 2020).

Em síntese, é perceptível que através da redução dos investimentos do SUS e com o aumento da procura de leitos em unidades hospitalares, onde muitas vezes há falta de profissionais de insumos e de estrutura adequada, gera um grande caos nos atendimentos principalmente nesse período pandêmico, isso só faz demonstrar a fragilidade do Sistema Único de Saúde, que apesar apresentar vários problemas de precarização de seus atendimentos, ainda é o lugar mais viável para a busca de atendimento médico, principalmente por uma grande maioria da população brasileira.

\section{Considerações Finais}

Diante das reflexões estudadas ao longo da pesquisa, foi possível chegar a um entendimento de que, apesar dos direitos fundamentais estarem consolidados na Constituição Federal de 1988, verifica-se que o Estado, não garante o suficiente para que se possa efetivar essas garantias, e assim é criado um ambiente social onde o sacrifício trona-se uma opção.

Em frente a esse descaso feito pelo estado, fica evidenciado principalmente nesse período de pandemia, a falta de um investimento que seja mais aplicado na saúde pública nacional e atrelado ao sucateamento do sistema possibilita um aprofundamento da necropolítica, a qual julga que o direito a saúde de grande parte da população é descartável, e nesse sentido inviabiliza o efetivo direito à vida, ao pôr em segundo plano a valorização do SUS.

Neste contexto, a falta de investimento adequado nesse sistema, além de possibilitar e acarretar uma defasagem das estruturas públicas, impõe o investimento em garantias como algo a ser realizado como um segundo plano, sendo de fácil percepção a forma que o sistema único de saúde vem sofrendo com a ausência de investimentos por parte do governo, para a promoção da igualdade a saúde.

Portanto, é preciso que diante dessa problemática, os governos, gestores e demais membros da sociedade, possam elaborar propostas para que haja o fortalecimento da gestão pública, para garantir a suficiência de recursos que sejam destinados a saúde. Pois essa busca econômica é essencial, para que se tenha recursos de custeio garantidos para o pleno funcionamento do SUS, aliada a dispositivos institucionais eficazes de coordenação de políticas, nos espaços nacional, estadual 
e regional, para que se possa aspirar, minimamente, a superação da crônica e danosa crise hospitalar, fortemente agudizada pela pandemia no Brasil.

\section{Referências}

Araújo, J. L., Oliveira, K. K. D., \& Freitas, R. J. M. (2020). In defense of the Unified Health System in the context of SARS-CoV-2 pandemic. Rev Bras Enferm. 2020;73(Suppl 2):e20200247. doi: http://dx.doi.org/10.1590/0034-7167-2020-0247.

Araújo, J. C. (2008). Ensaios Sobre Impactos da Constituição Federal de 1988 Na Sociedade Brasileira. Brasília: Câmara dos Deputados, Edições Câmara.

Bahia, C. J. A., \& Abujamra, A. C. P. (1998). A Justiciabilidade do direito fundamental à saúde: Concretização do princípio constitucional da dignidade da pessoa humana. Revista dos Tribunais. São Paulo: Editora Revista dos Tribunais, 2010. Ano 99, volume 892, fevereiro. Brasil. Constituição Federal. Brasília DF.

Brasil. (1990). Lei $n^{\circ} 8.080$ de 19 de Setembro de 1990. Dispõe sobre as condições para a promoção, proteção e recuperação da saúde, a organização e o funcionamento dos serviços correspondentes e dá outras providências. In: Diário Oficial da República Federativa do Brasil, Brasília, DF.

Bahia, F. (2012). Direito Fundamental à Saúde. [vídeo]. Saber Direito. TV Justiça. 2012. https://www.youtube.com/watch?v=nYy1SF3bjv0

Chioro, A. et al. (2021). Financiamento do SUS e garantia de direitos [livro eletrônico]: orientação técnica e produção de dados na garantia de direitos no contexto da pandemia Covid-19/ Laboratório de Saúde Coletiva; São Paulo: UNIVERSIDADE FEDERAL DE SÃ̃O PAULO. https://redeaps.org.br/wpcontent/uploads/2021/06/ebook.pdf

Campos, F. C. C. de, \& Canabrava, C. M. (2020). O Brasil na UTI: atenção hospitalar em tempos de pandemia. DOI: 10.1590/SciELOPreprints.1368

Servo, L. M. S., Santos, M. A. B. dos., Vieira, F. S., \& Benevides e Sá, R. P. de. (2020). Financiamento do SUS e Covid-19: histórico, participações federativas e respostas à pandemia SUS financing and Covid-19: history, federative participation, and responses to the pandemic DOI: 10.1590/0103$11042020 \mathrm{E} 407$

Maia, A. D. (2020). direito à saúde e a pandemia da covid-19: desafios para o estado democrático de direito brasileiro. Revista Processus de Estudos de Gestão, Jurídicos e Financeiros, 11(41). Doi: https://doi.org/10.5281/zenodo.4319208.

Mendes, A., \& Marques, R. (2010). A saúde pública sob a batuta da nova ordem. In: Marques, R., \& Ferreira, M. R. J., organização. O Brasil sob nova ordem: a economia brasileira contemporânea. São Paulo: Saraiva.

Moura, E. S. de. (2013). O direito à saúde na Constituição Federal de 1988. Revista Jus Navigandi, ISSN 1518-4862, Teresina, ano 18, n. 3730 , 17 set. 2013. https://jus.com.br/artigos/25309

Organização Pan-Americana da Saúde. (2013). Financiamento público de saúde [internet]. Brasília. https://bit.ly/3hfiQQu

Paim, J. S. (2018). Sistema Único de Saúde (SUS) aos 30 anos. Ciência \& Saúde Coletiva, 23(6). http://repositorio.ufba.br/ri/handle/ri/29600

Santos, L., \& Funcia, F.R. (2020). Histórico do financiamento do SUS: evidências jurídico-orçamentárias do desinteresse governamental federal sobre a garantia do direito fundamental à saúde. Doming. da Saúde. https://bit.ly/36pNN1D

Silva, W. M. F. da., Ruiz, J. L. de S. (2020). A centralidade do SUS na pandemia do coronavírus e as disputas com o projeto neoliberal. Physis: Revista de Saúde Coletiva 2020 journal-article DOI: https://doi.org/10.1590/S0103-73312020300302 